International Journal of Pure and Applied Mathematics

Volume 98 No. 1 2015, 65-79

ISSN: 1311-8080 (printed version); ISSN: 1314-3395 (on-line version)

url: http://www.ijpam.eu

doi: http://dx.doi.org/10.12732/ijpam.v98i1.7

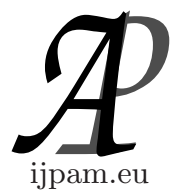

\title{
GENERALIZED HYERS-ULAM STABILITY OF REFINED QUADRATIC FUNCTIONAL EQUATIONS
}

\author{
Hark-Mahn Kim ${ }^{1}$, Hwan-Yong Shin ${ }^{2} \S$ \\ ${ }^{1,2}$ Department of Mathematics \\ Chungnam National University \\ Daejeon 305-764, REPUBLIC OF KOREA
}

\begin{abstract}
In this paper, we give a general solution of a refined quadratic functional equation and then investigate its generalized Hyers-Ulam stability in quasi-normed spaces and in non-Archimedean normed spaces.
\end{abstract}

AMS Subject Classification: 39B82, 39B62

Key Words: quasi-normed spaces, $p$-normed spaces, non-Archimedean normed spaces

\section{Introduction}

In [28], S.M. Ulam proposed the stability problem for functional equations concerning the stability of group homomorphisms. A functional equation is called stable if any approximate solution to the functional equation is near a true solution of that functional equation. In [12], D.H. Hyers considered the case of approximate additive mappings with the Cauchy difference controlled by a positive constant in Banach spaces. D.G. Bourgin [5] and T. Aoki [2] treated this problem for approximate additive mappings controlled by unbounded functions. In [23], Th. M. Rassias provided a generalization of Hyers' theorem for linear mappings which allows the Cauchy difference to be unbounded. In 1994, P. Găvruta [9] generalized these theorems for approximate additive mappings controlled by the unbounded Cauchy difference with regular conditions. During

Received: August 28, 2014

(c) 2015 Academic Publications, Ltd.

${ }^{\S}$ Correspondence author url: www.acadpubl.eu 
the last three decades a number of papers and research monographs have been published on various generalizations and applications of the Hyers-Ulam stability and generalized Hyers-Ulam stability to a number of functional equations and mappings $[1,6,8,13,22]$.

A stability problem of Ulam for the quadratic functional equation

$$
f(x+y)+f(x-y)=2 f(x)+2 f(y)
$$

was first proved by $\mathrm{F}$. Skof for mapping $f: E_{1} \rightarrow E_{2}$, where $E_{1}$ is a normed space and $E_{2}$ is a Banach space [26]. In the paper [7], S. Czerwik proved the Hyers-Ulam-Rassias stability of the quadratic functional equation (1). In particular, J.M. Rassias $[19,20,21]$ solved the stability problem of Ulam for the Euler-Lagrange type quadratic functional equation

$$
f(r x+s y)+f(s x-r y)=\left(r^{2}+s^{2}\right)[f(x)+f(y)]
$$

for fixed real numbers $r, s$ with $r \neq 0, s \neq 0$.

In particular, P.L. Kannappan [15] introduced the following functional equation

$$
\begin{aligned}
& f(x+y)+f(y+z)+f(z+x) \\
& =f(x+y+z)+f(x)+f(y)+f(z)
\end{aligned}
$$

and proved that a function on a real vector space is a solution of (3) if and only if there exist a symmetric biadditive function $B$ and an additive function $A$ such that $f(x)=B(x, x)+A(x)$. In [3], the authors proved the generalized Hyers-Ulam stability of the functional equation

$$
\begin{aligned}
& f(x-y)+f(y-z)+f(z-x)+f(x+y+z) \\
& =3[f(x)+f(y)+f(z)]
\end{aligned}
$$

which is equivalent to the quadratic equation (1). Recently, Zivari-Kazempour and M. Eshaghi Gordji [29] proved the general solution of the following quadratic functional equation

$$
\begin{aligned}
& f(x+2 y)+f(y+2 z)+f(z+2 x) \\
& =2 f(x+y+z)+3[f(x)+f(y)+f(z)]
\end{aligned}
$$

and investigated the Hyers-Ulam stability of the equation (5) in Banach space.

In this paper, we consider the following functional equation

$$
f(x+n y)+f(y+n z)+f(z+n x)
$$




$$
=n f(x+y+z)+\left(n^{2}-n+1\right)[f(x)+f(y)+f(z)],
$$

where $n$ is any fixed nonzero integer, and then investigate the Hyers-Ulam stability of the equation (6).

\section{General Solution}

First, we remark that the equation (6) is equivalent to (4) for the case $n=-1$ [3], and the equation (6) is trivially equivalent to (3) for the case $n=1$. Thus we give the general solution of the equation (6) for the case $n \neq-1,0,1$ in the following Theorem 2.3.

Lemma 1. Let $X$ and $Y$ be vector spaces and $f: X \rightarrow Y$ be an even function satisfying the functional equation (6). Then $f$ is quadratic.

Proof. Assume that a function $f: X \rightarrow Y$ satisfies (6). Letting $y=z:=x$ in (6), we get

$$
3 f((n+1) x)=n f(3 x)+3\left(n^{2}-n+1\right) f(x)
$$

for all $x \in X$, which implies $f(0)=0$. Letting $y=z:=0$ in (6), we have

$$
f(n x)=n^{2} f(x)
$$

for all $x \in X$. Putting $z:=0, y:=x$ in (6) and using (8), then we get

$$
f((n+1) x)=n f(2 x)+(n-1)^{2} f(x)
$$

for all $x \in X$. Combining (7) and (9), we obtain

$$
3 f(2 x)=f(3 x)+3 f(x)
$$

for all $x \in X$. Letting $(x, y, z):=(-x, x, 0)$ in $(6)$, we have

$$
f((n-1) x)=\left(n^{2}-n\right) f(x)+(-n+1) f(-x)
$$

for all $x \in X$. Replacing $x$ by $-x$ in (11) and adding it with (11), we get

$$
f((n-1) x)+f(-(n-1) x)=(n-1)^{2}[f(-x)+f(x)]
$$

for all $x \in X$. Letting $y:=x, z:=-x$ in (6) and using (9) and (12), we get

$$
f(2 x)=3 f(x)+f(-x)
$$


for all $x \in X$. Combining (10) and (13), we get

$$
f(3 x)=6 f(x)+3 f(-x)
$$

for all $x \in X$.

Now, suppose that $f$ is an even function satisfying (6). Then we get $f((n-$ $1) x)=(n-1)^{2} f(x)$ by $(12)$ and $f((n+1) x)=(n+1)^{2} f(x)$ by (9). Putting $z:=-y$ in $(6)$, we get

$$
f(x+n y)+f(n x-y)=\left(n^{2}+1\right)[f(x)+f(y)]
$$

for all $x, y \in X$. Letting $z:=0$ in (6), we have

$$
f(x+n y)=n f(x+y)+(-n+1) f(x)+\left(n^{2}-n\right) f(y)
$$

for all $x, y \in X$. Exchanging $x$ and $y$ in (16), we obtain

$$
f(n x+y)=n f(x+y)+(-n+1) f(y)+\left(n^{2}-n\right) f(x)
$$

for all $x, y, \in X$. Replacing $y$ by $-y$ in (17), we arrive at

$$
f(n x-y)=n f(x-y)+(-n+1) f(y)+\left(n^{2}-n\right) f(x)
$$

for all $x, y \in X$. Applying (16) and (18) to (15), we get the desired equation

$$
f(x+y)+f(x-y)=2 f(x)+2 f(y)
$$

for all $x, y \in X$. Therefore $f$ is quadratic.

Lemma 2. If $f: X \rightarrow Y$ is an odd function satisfying (6), then $f \equiv 0$.

Proof. We get $f(2 x)=2 f(x)$ and $f(3 x)=3 f(x)$ from (13) and (14), respectively. Using these equations, we have $f((n+1) x)=\left(n^{2}+1\right) f(x)$ and $f((n-1) x)=\left(n^{2}-1\right) f(x)$ from (9) and (11), respectively. Putting $z:=-y$ in (6), we get

$$
f(x+n y)+f(n x-y)=\left(n^{2}+1\right) f(x)+\left(n^{2}-1\right) f(y)
$$

for all $x, y \in X$. Letting $z:=0$ in (6), we have

$$
f(x+n y)=n f(x+y)+(-n+1) f(x)+\left(n^{2}-n\right) f(y)
$$

for all $x, y \in X$. Taking $x:=-y$ and $y:=x$ in (20), we obtain

$$
f(n x-y)=n f(x-y)+(-n+1) f(y)+\left(n^{2}-n\right) f(x)
$$


for all $x, y \in X$. Applying (20) and (21) to (19), then we get

$$
f(x+y)+f(x-y)=2 f(x)
$$

for all $x, y \in X$. Thus, $f$ is additive and so $f(n x)=n f(x)$ for all $x \in X$. Associating the last equation with (8), we get $f \equiv 0$.

Theorem 3. Let $f: X \rightarrow Y$ be a function satisfying the functional equation (6). Then $f$ is quadratic and so (6) is equivalent to (1) for the case $n \neq-1,0,1$.

Proof. We can express $f(x)=f_{e}(x)+f_{o}(x)$, where $f_{e}(x)=\frac{f(x)+f(-x)}{2}$ is even and $f_{o}(x)=\frac{f(x)-f(-x)}{2}$ is odd. Thus one can easily find that $f_{e}$ and $f_{o}$ satisfy the equation (6). Therefore, $f_{o} \equiv 0$ and so $f=f_{e}$ is quadratic and so the equation is equivalent to (1).

\section{The Hyers-Ulam Stability in Quasi-Banach Spaces}

In this section, we investigate the generalized Hyers-Ulam stability problem for the functional equation (6) in quasi-Banach space. First, we introduce some basic information concerning quasi-Banach spaces which are referred in [4] and [25]. Let $X$ be a linear space. A quasi-norm is a real-valued function on $X$ satisfying the following:

(i) $\|x\| \geq 0$ for all $x \in X$, and $\|x\|=0$ if and only if $x=0$;

(ii) $\|\lambda x\|=|\lambda|\|x\|$ for any scalar $\lambda$ and all $x \in X$;

(iii) There is a constant $M \geq 1$ such that $\|x+y\| \leq M(\|x\|+\|y\|)$ for all $x, y \in X$.

The pair $(X,\|\cdot\|)$ is called a quasi-normed space if $\|\cdot\|$ is a quasi-norm on $X$. The smallest possible $M$ is called the modulus of concavity of the quasi-norm $\|\cdot\|$. A quasi-Banach space is a complete quasi-normed space. A quasi-norm $\|\cdot\|$ is called a $q$-norm $(0<q \leq 1)$ if $\|x+y\|^{q} \leq\|x\|^{q}+\|y\|^{q}$ for all $x, y \in X$. In this case, a quasi-Banach space is called a $q$-Banach space. Let $X$ be a quasi-Banach space. Given a $q$-norm, the formula $d(x, y):=\|x-y\|^{q}$ gives us a translation invariant metric on X. By Aoki-Rolewicz Theorem [25] (see also [4]), each quasi-norm is equavalent to some $q$-norm. Since it is much easier 
to work with $q$-norms than quasi-norms, here and subsequently, we restrict our attention mainly to $q$-norms. Moreover, generalized stability theorems of functional equations in quasi-Banach spaces have been investigated by a lot of authors [14, 18, 27].

Now we introduce an abbreviation $D_{n} f$ for a given mapping $f: X \rightarrow Y$ as follows:

$$
\begin{aligned}
D_{n} f(x, y, z):= & f(x+n y)+f(y+n z)+f(z+n x) \\
& -n f(x+y+z)-\left(n^{2}-n+1\right)[f(x)+f(y)+f(z)]
\end{aligned}
$$

for all $x, y, z \in X$, where $n \neq-1,0,1$ is a fixed integer.

From now on, let $X$ be a normed linear space with norm $\|\cdot\|$ and $Y$ be a $q$-Banach space with norm $\|\cdot\|$. In this part, by using an direct method, we prove the stability theorem of the equation (6).

Theorem 4. Let $\phi: X^{3} \rightarrow[0, \infty)$ be a function such that

$$
\sum_{j=0}^{\infty} \frac{1}{n^{2 j q}} \phi\left(n^{j} x, 0,0\right)^{q}<\infty, \quad \lim _{j \rightarrow \infty} \frac{\phi\left(n^{j} x, n^{j} y, n^{j} z\right)}{n^{2 j}}=0
$$

for all $x, y, z \in X$. Suppose that a mapping $f: X \rightarrow Y$ with $f(0)=0$ satisfies the inequality

$$
\left\|D_{n} f(x, y, z)\right\| \leq \phi(x, y, z)
$$

for all $x, y, z \in X$. Then there exists a unique quadratic mapping $Q: X \rightarrow Y$ such that

$$
\|f(x)-Q(x)\| \leq \frac{1}{n^{2}}\left[\sum_{j=0}^{\infty} \frac{\phi\left(n^{j} x, 0,0\right)^{q}}{n^{2 j q}}\right]^{\frac{1}{q}}
$$

for all $x \in X$.

Proof. Replacing $(x, y, z)$ by $(x, 0,0)$ in $(24)$, we have

$$
\left\|f(n x)-n^{2} f(x)\right\| \leq \phi(x, 0,0)
$$

for all $x \in X$. Replacing $x$ by $n^{k} x$ in (26) and then dividing both sides by $n^{2 k+2}$, we get

$$
\left\|\frac{1}{n^{2 k}} f\left(n^{k} x\right)-\frac{1}{n^{2 k+2}} f\left(n^{k+1} x\right)\right\| \leq \frac{1}{n^{2}} \frac{\phi\left(n^{k} x, 0,0\right)}{n^{2 k}}
$$


for all $x \in X$ and all integers $k \geq 0$. Then for any integers $m, k$ with $m \geq k \geq 0$, we obtain

$$
\begin{aligned}
& \left\|\frac{1}{n^{2 m+2}} f\left(n^{m+1} x\right)-\frac{1}{n^{2 k}} f\left(n^{k} x\right)\right\|^{q} \\
& =\left\|\sum_{j=k}^{m}\left(\frac{1}{n^{2 j+2}} f\left(n^{j+1} x\right)-\frac{1}{n^{2 j}} f\left(n^{j} x\right)\right)\right\|^{q} \\
& \leq \sum_{j=k}^{m}\left\|\frac{1}{n^{2 j+2}} f\left(n^{j+1} x\right)-\frac{1}{n^{2 j}} f\left(n^{j} x\right)\right\|^{q} \\
& \leq \frac{1}{n^{2 q}} \sum_{j=k}^{m} \frac{\phi\left(n^{j} x, 0,0\right)^{q}}{n^{2 j q}}
\end{aligned}
$$

for all $x \in X$. Thus the sequence $\left\{\frac{f\left(n^{k} x\right)}{n^{2 k}}\right\}_{k=1}^{\infty}$ is Cauchy by (23). Since $Y$ is complete, this sequence converges for all $x \in X$. So one can define a mapping $Q: X \rightarrow Y$ by

$$
\lim _{k \rightarrow \infty} \frac{f\left(n^{k} x\right)}{n^{2 k}}=Q(x) \quad(x \in X) .
$$

It follows from (23) and (28) that

$$
\begin{aligned}
\left\|D_{n} Q(x, y, z)\right\| & =\lim _{k \rightarrow \infty} \frac{1}{n^{2 k}}\left\|D_{n} f\left(n^{k} x, n^{k} y, n^{k} z\right)\right\| \\
& \leq \lim _{k \rightarrow \infty} \frac{\phi\left(n^{k} x, n^{k} y, n^{k} z\right)}{n^{2 k}}=0
\end{aligned}
$$

for all $x, y, z \in X$. Hence, the mapping $Q$ satisfies (6) and so it is quadratic. Putting $k:=0$ and letting $m$ go to infinity in (27), we see that (25) holds. For the uniqueness of $Q$, assume that there exists a quadratic mapping $Q^{\prime}: X \rightarrow Y$ satisfying the inequality (25). Then, we find that

$$
\begin{aligned}
\left\|Q(x)-Q^{\prime}(x)\right\|^{q} & =\lim _{k \rightarrow \infty} \frac{1}{n^{2 k q}}\left\|f\left(n^{k} x\right)-Q^{\prime}\left(n^{k} x\right)\right\|^{q} \\
& \leq \lim _{k \rightarrow \infty} \frac{1}{n^{2 q} n^{2 k q}} \sum_{j=0}^{\infty} \frac{1}{n^{2 j q}} \phi\left(n^{j+k} x, 0,0\right)^{q} \\
& =\frac{1}{n^{2 q}} \lim _{k \rightarrow \infty} \sum_{j=k}^{\infty} \frac{1}{n^{2 k q}} \phi\left(n^{k} x, 0,0\right)^{q}=0
\end{aligned}
$$

for all $x \in X$, which proves the uniqueness. 
Theorem 5. Let $\phi: X^{3} \rightarrow[0, \infty)$ be a function such that

$$
\sum_{j=0}^{\infty} n^{2 j q} \phi\left(n^{-j} x, 0,0\right)^{q}<\infty, \quad \lim _{j \rightarrow \infty} n^{2 j} \phi\left(n^{-j} x, n^{-j} y, n^{-j} z\right)=0
$$

for all $x, y, z \in X$. Suppose that $f: X \rightarrow Y$ is a mapping with $f(0)=0$ satisfying the inequality

$$
\left\|D_{n} f(x, y, z)\right\| \leq \phi(x, y, z)
$$

for all $x, y, z \in X$. Then there exists a unique quadratic mapping $Q: X \rightarrow Y$ such that

$$
\|f(x)-Q(x)\| \leq \frac{1}{n^{2}}\left[\sum_{j=1}^{\infty} n^{2 j q} \phi\left(n^{-j} x, 0,0\right)^{q}\right]^{\frac{1}{q}}
$$

for all $x \in X$.

Proof. We observe that one can obtain the following inequality

$$
\left\|n^{2 k} f\left(\frac{x}{n^{k}}\right)-n^{2(m+1)} f\left(\frac{x}{n^{m+1}}\right)\right\|^{q} \leq \frac{1}{n^{2 q}} \sum_{j=k}^{m} n^{2(j+1) q} \phi\left(n^{-(j+1)} x, 0,0\right)^{q}
$$

for all $x \in X$ and all integers $k, m$ with $m \geq k \geq 0$ by use of (26). Thus, we see that the proof may be verified by applying similar argument to that of Theorem 4.

Corollary 6. Let $\varepsilon \geq 0$. Suppose that a mapping $f: X \rightarrow Y$ with $f(0)=0$ satisfies the inequality

$$
\left\|D_{n} f(x, y, z)\right\| \leq \varepsilon
$$

for all $x, y, z \in X$. Then there exists a unique quadratic mapping $Q: X \rightarrow Y$ such that

$$
\|f(x)-Q(x)\| \leq \frac{\varepsilon}{\sqrt[q]{n^{2 q}-1}}
$$

for all $x \in X$. 
Corollary 7. Let $\alpha, a_{1}, a_{2}, a_{3}$ be positive real numbers such that either $a_{i}>2$ or $a_{i}<2$ for all $i \in\{1,2,3\}$. Suppose that a mapping $f: X \rightarrow Y$ with $f(0)=0$ satisfies the inequality

$$
\left\|D_{n} f(x, y, z)\right\| \leq \alpha\left(\|x\|^{a_{1}}+\|y\|^{a_{2}}+\|z\|^{a_{3}}\right)
$$

for all $x, y, z \in X$. Then there exists a unique quadratic mapping $Q: X \rightarrow Y$ such that

$$
\|f(x)-Q(x)\| \leq \frac{\alpha\|x\|^{a_{1}}}{\sqrt[q]{\mid n^{2 q}-n^{q \cdot a_{1} \mid}}}
$$

for all $x \in X$.

\section{The Hyers-Ulam Stability in Non-Archimedean Spaces}

Hensel [11] has introduced a normed space which does not have the nonArchimedean spaces property. During the last three decades, the theory of non-Archimedean spaces has gain the interest of physicists for their research in problems coming from quantum physics, $p$-adic strings and superstrings [16].

A valuation is a function $|\cdot|$ from a field $\mathbb{K}$ to $[0, \infty)$ such that 0 is the unique element having the 0 valuation, $|r s|=|r| \cdot|s|$ and the triangle inequality holds, i.e.,

$$
|r+s| \leq|r|+|s|, \forall r, s \in \mathbb{K} .
$$

A field $\mathbb{K}$ is called a valued field if $\mathbb{K}$ equips with a valuation. The usual absolute values of $\mathbb{R}$ and $\mathbb{C}$ are examples of valuations. Alternatively, if the triangle inequality is replaced by the strong triangle inequality

$$
|r+s| \leq \max \{|r|,|s|\}, \forall r, s \in \mathbb{K},
$$

then the valuation $|\cdot|$ is called a non-Archimedean valuation, and the field is called a non-Archimedean field. Cleary $|1|=|-1|=1$ and $|n| \leq 1$ for all $n \in \mathbb{N}$. A trivial example of a non-Archimedean valuation is the function $|\cdot|$ taking everything except for 0 into 1 and $|0|=0$.

Definition 8. Let $X$ be a vector space over a field $\mathbb{K}$ with a nonArchimedean valuation $|\cdot|$. A function $\|\cdot\|: X \rightarrow[0, \infty)$ is said to be a non-Archimedean norm on $\mathrm{X}$ if it satisfies the following conditions

(i) $\|x\|=0$ if and only if $x=0$; 
(ii) $\|r x\|=|r|\|x\|(r \in \mathbb{K})$;

(iii) $\|x+y\| \leq \max \{\|x\|,\|y\|\} \quad(x, y \in X)$.

In this case $(X,\|\cdot\|)$ is called a non-Archimedean normed space. Because of the fact

$$
\left\|x_{k}-x_{m}\right\| \leq \max \left\{\left\|x_{j+1}-x_{j}\right\|: m \leq j \leq k-1\right\} \quad(k>m),
$$

a sequence $\left\{x_{m}\right\}$ is Cauchy in the non-Archimedean normed space if and only if $\left\{x_{m+1}-x_{m}\right\}$ converges to zero with respect to the non-Archimedean norm. By a complete non-Archimedean space we mean one in which every Cauchy sequence is convergent.

Example 9. Let $p$ be a prime number. For any nonzero rational number $x$, there exists a unique integer $n_{x} \in \mathbb{Z}$ such that $x=\frac{a}{b} p^{n_{x}}$, where $a$ and $b$ are integers not divisible by $p$. Then $|x|_{p}:=p^{-n_{x}}$ defines a non-Archimedean norm on $\mathbb{Q}$. The completion of $\mathbb{Q}$ with respect to the metric $d(x, y)=|x-y|_{p}$ is denoted by $\mathbb{Q}_{p}$ which is called the $p$-adic number field. In fact, $\mathbb{Q}_{p}$ is the set of all formal series $x=\sum_{k \geq n_{x}}^{\infty} a_{k} p^{k}$, where $\left|a_{k}\right| \leq p-1$ are integers. The addition and multiplication between any two elements of $\mathbb{Q}_{p}$ are defined naturally. The norm $\left|\sum_{k \geq n_{x}}^{\infty} a_{k} p^{k}\right|=p^{-n_{x}}$ is a non-Archimedean norm on $\mathbb{Q}_{p}$ and it makes $\mathbb{Q}_{p}$ a locally compact field (see $[10,24]$ ).

Let $X$ be a vector space and $Y$ be a non-Archimedean Banach space. In the following, we now prove the generalized Hyers-Ulam stability of quadratic functional equation (6) over the non-Archimedean space. As corollaries, we obtain especially stability result over the $p$-adic field $\mathbb{Q}_{p}$. To avoid trivial case, we assume $|n|<1$. that

Theorem 10. Let $\phi: X^{3} \rightarrow[0, \infty)\left(\psi: X^{3} \rightarrow[0, \infty)\right)$ be a function such

$$
\begin{aligned}
& \lim _{j \rightarrow \infty} \frac{\phi\left(n^{j} x, n^{j} y, n^{j} z\right)}{|n|^{2 j}}=0 \\
& \left(\lim _{j \rightarrow \infty}|n|^{2 j} \psi\left(n^{-j} x, n^{-j} y, n^{-j} z\right)=0, \text { resp }\right)
\end{aligned}
$$

for all $x, y, z \in X$ and the limit

$$
\Phi(x) \equiv \lim _{k \rightarrow \infty} \max \left\{\frac{\phi\left(n^{j} x, 0,0\right)}{|n|^{2 j}}: 0 \leq j<k\right\}
$$




$$
\left(\Psi(x) \equiv \lim _{k \rightarrow \infty} \max \left\{|n|^{2 j} \psi\left(n^{-j} x, 0,0\right): 1 \leq j \leq k\right\}, \text { resp }\right)
$$

exists for each $x \in X$. Suppose that a mapping $f: X \rightarrow Y$ with $f(0)=0$ satisfies the inequality

$$
\begin{aligned}
& \left\|D_{n} f(x, y, z)\right\| \leq \phi(x, y, z) \\
& \left(\left\|D_{n} f(x, y, z)\right\| \leq \psi(x, y, z), \text { resp }\right),
\end{aligned}
$$

for all $x, y, z \in X$. Then there exists a quadratic mapping $Q: X \rightarrow Y$ such that

$$
\begin{aligned}
& \|f(x)-Q(x)\| \leq \frac{1}{|n|^{2}} \Phi(x) \\
& \left(\|f(x)-Q(x)\| \leq \frac{1}{|n|^{2}} \Psi(x), \text { resp }\right),
\end{aligned}
$$

for all $x \in X$. Moreover, if

$$
\begin{aligned}
& \lim _{m \rightarrow \infty} \lim _{k \rightarrow \infty} \max \left\{\frac{\phi\left(n^{j} x, 0,0\right)}{|n|^{2 j}}: m \leq j<k+m\right\}=0 \\
& \left(\lim _{m \rightarrow \infty} \lim _{k \rightarrow \infty} \max \left\{|n|^{2 j} \psi\left(n^{-j} x, 0,0\right): m<j \leq k+m\right\}=0, \text { resp }\right),
\end{aligned}
$$

for all $x \in X$, then the quadratic mapping $Q$ is unique.

Proof. Replacing $(x, y, z)$ by $(x, 0,0)$ in $(31)$, we have

$$
\left\|f(n x)-n^{2} f(x)\right\| \leq \phi(x, 0,0)
$$

for all $x \in X$. Replacing $x$ by $n^{k} x$ in (34) and then dividing both sides by $|n|^{2 k+2}$, we get

$$
\left\|\frac{1}{n^{2 k+2}} f\left(n^{k+1} x\right)-\frac{1}{n^{2 k}} f\left(n^{k} x\right)\right\| \leq \frac{1}{|n|^{2}} \frac{\phi\left(n^{k} x, 0,0\right)}{|n|^{2 k}}
$$

for all $x \in X$. It follows from (35) and (29) that the sequence $\left\{\frac{f\left(n^{k} x\right)}{n^{2 k}}\right\}_{k=1}^{\infty}$ is Cauchy in the non-Archimedean normed space Y. Since $Y$ is complete, we may define a mapping $Q: X \rightarrow Y$ as $Q(x):=\lim _{k \rightarrow \infty} \frac{f\left(n^{k} x\right)}{n^{2 k}}$ for all $x \in X$. Using induction, one can show that

$$
\left\|\frac{f\left(n^{k} x\right)}{n^{2 k}}-f(x)\right\| \leq \frac{1}{|n|^{2}} \max \left\{\frac{\phi\left(n^{j} x, 0,0\right)}{|n|^{2 j}}: 0 \leq j<k\right\}
$$


for all $k \in \mathbb{N}$ and all $x \in X$. By taking $k$ to approach infinity in (36) and using (30), one obtains (32). Replacing $x, y$ and $z$ by $n^{2 k} x, n^{2 k} y$ and $n^{2 k} z$, respectively, in (31), we get

$$
\left\|\frac{D_{n} f\left(n^{k} x, n^{k} y, n^{k} z\right)}{n^{2 k}}\right\| \leq \frac{\phi\left(n^{k} x, n^{k} y, n^{k} z\right)}{|n|^{2 k}}
$$

for all $x, y, z \in X$. Taking the limit as $k \rightarrow \infty$ and using Theorem 3, we conclude that $Q$ is quadratic. Moreover, to prove the uniqueness, we assume that there exists a quadratic mapping $Q^{\prime}: X \rightarrow Y$ satisfying (32) and (33). Then we figure out

$$
\begin{aligned}
& \left\|Q(x)-Q^{\prime}(x)\right\| \\
& =\lim _{m \rightarrow \infty} \frac{1}{|n|^{2 m}}\left\|Q\left(n^{m} x\right)-Q^{\prime}\left(n^{m} x\right)\right\| \\
& \leq \lim _{m \rightarrow \infty} \max \left\{\frac{\left\|Q\left(n^{m} x\right)-f\left(n^{m} x\right)\right\|}{|n|^{2 m}}, \frac{\left\|f\left(n^{m} x\right)-Q^{\prime}\left(n^{m} x\right)\right\|}{|n|^{2 m}}\right\} \\
& \leq \lim _{m \rightarrow \infty} \lim _{k \rightarrow \infty} \frac{1}{|n|^{2}} \max \left\{\frac{\phi\left(n^{j} x, 0,0\right)}{|n|^{2 j}}: m \leq j<m+k\right\}=0
\end{aligned}
$$

for all $x \in X$. This completes the proof.

Corollary 11. Let $X$ be a non-Archimedean normed space, $r \neq 2$ and $\varepsilon, \theta$ be positive numbers, where $\varepsilon=0$ if $r>2$. Suppose that a mapping $f: X \rightarrow Y$ with $f(0)=0$ satisfies the inequality

$$
\left\|D_{n} f(x, y, z)\right\| \leq \varepsilon+\theta\left(\|x\|^{r}+\|y\|^{r}+\|z\|^{r}\right) \quad(x, y, z \in X) .
$$

Then there exists a unique quadratic mapping $Q: X \rightarrow Y$ such that

$$
\|f(x)-Q(x)\| \leq\left\{\begin{array}{lll}
\varepsilon+\frac{\theta}{|n|^{r}}\|x\|^{r} & \text { if } & r<2 \\
\frac{\theta}{|n|^{2}}\|x\|^{r} & \text { if } & r>2
\end{array}\right.
$$

for all $x \in X$.

Corollary 12. Let $r \neq 2$ and $\varepsilon, \theta$ be positive numbers, where $\varepsilon=0$ if $r>2$. Suppose that a mapping $f: \mathbb{Q}_{p} \rightarrow \mathbb{Q}_{p}$ with $f(0)=0$ satisfies the inequality

$$
\left|D_{p} f(x, y, z)\right|_{p} \leq \varepsilon+\theta\left(|x|_{p}^{r}+|y|_{p}^{r}+|z|_{p}^{r}\right) \quad\left(x, y, z \in \mathbb{Q}_{p}\right) .
$$


Then there exists a unique quadratic mapping $Q: \mathbb{Q}_{p} \rightarrow \mathbb{Q}_{p}$ such that

$$
|f(x)-Q(x)|_{p} \leq\left\{\begin{array}{lll}
\varepsilon+p^{r} \theta|x|_{p}^{r} & \text { if } & r<2 \\
p^{2} \theta|x|_{p}^{r} & \text { if } & r>2
\end{array}\right.
$$

for all $x \in \mathbb{Q}_{p}$.

\section{Acknowledgements}

This research was supported by Basic Research Program through the National Research Foundation of Korea (NRF) funded by the Ministry of Education (NRF-2012R1A1A2008139).

\section{References}

[1] J. Aczel and J. Dhombres, Functional Equations in Several Variables, Cambridge University Press, (1989), Doi:10.1017/CBO9781139086578.

[2] T. Aoki, On the stability of the linear transformation in Banach spaces, $J$. Math. Soc. Japan, 2, No. 1-2 (1950), 64-66, Doi:10.2969/jmsj/00210064.

[3] J-H. Bae and I-S. Chang, On the Ulam stability problem of a quadratic functional equation, Korean J. Comput, Appl. Math.(Series A), 8, No. 2 (2001), 561-567.

[4] Y. Benyamini and J. Lindenstrauss, Geometric Nonlinear Functional Analysis, American Mathematical Society, (2000).

[5] D.G. Bourgin, Classes of transformations and bordering transformations, Bull. Amer. Math. Soc. 57 (1951), 223-237, Doi:10.1090/S0002-9904-195109511-7.

[6] Y-J. Cho, Th. M. Rassias, R. Saadati, Stability of Functional Equations in Random Normed Spaces, Springer Optimization and Its Applications, 86, Springer, (2013), Doi:10.1007/978-1-4614-8477-6.

[7] S. Czerwik, On the stability of the quadratic mapping in normed spaces, Bull. Abh. Math. Sem. Univ. Hamburg, 62 (1992), 59-64, Doi:10.1007/BF02941618. 
[8] S. Czerwik, Stability of Functional Equations of Ulam-Hyers-Rassias Type, Hadronic Press, Florida, (2003).

[9] P. Găvruta, A generalization of the Hyers-Ulam-Rassias stability of approximately additive mappings, J. Math. Anal. Appl. 184 (1994), 431-436, Doi:10.1006/jmaa.1994.1211.

[10] F. Q. Gouvêa, p-adic Numbers, Springer-Verlag, Berlin, (1997), Doi:10.1007/978-3-642-59058-04.

[11] K. Hensel, Über eine neue Begrndüng der Theorie der algebraischen Zahlen, Jahresber. Deutsch. Math. Verein, 6 (1987), 83-88.

[12] D. H. Hyers, On the stability of the linear functional equation, Proc. Nat. Acad. Sci. 27 (1941), 222-224, Doi:10.1073/pnas.27.4.222.

[13] S-M. Jung, Hyers-Ulam-Rassias Stability of Functional Equations in Nonlinear Analysis, Springer Optimization and Its Applications, USA, (2011), Doi:10.1007/978-1-4419-9637-4.

[14] K-W. Jun and H-M. Kim, On the stability of Euler-Lagrange type cubic mappings in quasi-Banach spaces, J. Math. Anal. Appl. 332 (2) (2006), 1335-1350, Doi:10.1016/j.jmaa.2006.11.024.

[15] Pl. Kannappan, Quadratic functional equation and inner product spaces, Results Math. 27 (1995), 368-372, Doi:10.1007/BF03322841.

[16] H. Khodaei and Th. M. Rassias, Approximately generalized additive functions in several variables, Int. J. Nonlinear Anal. Appl. 1 (2010), 2241.

[17] M. Mirzavaziri and M. S. Moslehian, A fixed point approach to stability of a quadratic equation, Bull. Brazilian Math. Soc. 37 (2006), 361-376, Doi:10.1007/s00574-006-0016-z.

[18] C. Park, Hyers-Ulam-Rassias stability of homomorphisms in quasi-Banach algebras, Bull. Sci. Math. 132(2) (2008), 87-96, Doi:10.1016/j.bulsci.2006.07.004.

[19] J.M. Rassias, On the stability of the Euler-Lagrange functional equation, Chinese J. Math. 20 (1992), 185-190. 
[20] J.M. Rassias, On the stability of the non-linear Euler-Lagrange functional equation in real normed linear spaces, J. Math. Phys. Sci. 28 (1994), 231235.

[21] J.M. Rassias, On the stability of the general Euler-Lagrange functional equation, Demonst. Math. 29 (1996), 755-766.

[22] Th. M. Rassias, Functional Equations, Inequalities and Applications, Kluwer Academic Publishers, Dordrecht, Boston and London (2003), Doi:10.1007/978-94-017-0225-6.

[23] Th. M. Rassias, On the stability of the linear mapping in Banach spaces, Proc. Amer. Math. Soc. 72 (1978), 297-300, Doi:10.1090/S0002-9939-19780507327-1.

[24] A. M. Robert, A Course in p-adic Analysis, Springer-Verlag, New-York, (2000), Doi:10.1007/978-1-4757-3254-2.

[25] S. Rolewicz, Metric linear spaces, Second edition. PWN-Polish Scientific Publishers, Warsaw:D. Reidel Publishing Co. Dordrecht, (1984).

[26] F. Skof, Local properties and approximations of operators, Rend. Sem. Math. Fis. Milano, 53 (1983), 113-129.

[27] J. Tabor, Stability of the Cauchy functional equation in quasi-Banach spaces, Ann. Polon. Math. 83 (2004), 243-255, Doi:10.4064/ap83-3-6.

[28] S. M. Ulam, Problems in Modern Mathematics, Chapter 6, Wiley Interscience, New York, (1964).

[29] A. Zivari-Kazempour and M. Eshaghi Gordji, Generalized Hyers-Ulam stabilities of an Euler-Lagrange-Rassias quadratic functional equation, Asian-European Journal of Mathematics, 5 (2012), Doi:10.1142/S1793557112500143. 
